\title{
Integration of Cables in the Virtual Product Development Process
}

\author{
Elke Hergenröther and Stefan Müller \\ Fraunhofer Institute für Graphische Datenverarbeitung $e$. $V$.
}

\begin{abstract}
In car-and aircraft industry virtual environments became an important technology in the product development process. The goal is to replace the physical mock-ups step by step with digital mock-ups. Using digital mock-ups the development time and the mock-up production costs can be reduced. The assumption is that digital mock-ups should have the same functionality as the physical mock-ups. Today bendable elements like cables, pipes or rubber plugs are not integrated in the virtual prototype development process. In this paper we analyse the integration of virtual cables in the digital mock-up development process. We also present a prototypical application in a virtual environment (VE), which one can use for the installation of a wiring harness and for assembly.
\end{abstract}

Key words: manufacturing, virtual prototyping, user interface for virtual environments

\section{INTRODUCTION}

In both the car- and the aircraft industry virtual environments have become an important technology in the product development process. The goal is to replace the physical mock-ups step by step with digital mock-ups. Using digital mock-ups reduces both the development time and the mock-up production costs. The assumption is that digital mock-ups should have the same functionality as the physical mock-ups. Therefore, the integration of cables, pipes and rubber plugs as bendable elements in the used applications is necessary. Until today these types of elements are not sufficiently integrated in the virtual prototype development process. To compensate these disadvantages we developed a cable installation and manipulation application in a virtual environment. This application can be used for the

The original version of this chapter was revised: The copyright line was incorrect. This has been corrected. The Erratum to this chapter is available at DOI: 10.1007/978-0-387-35492-7_50 
installation of a cable harness and for the assembly and disassembly simulation.

\section{ANALYSE OF THE ACTUAL SITUATION IN THE DEVELOPMENT PROCESS}

The construction phase of a car can be divided in two different parts. At first the mock-up is designed in the design phase. Here the shape of the car or the aircraft is fixed and it is planed how much space the single elements like motor, air filter and so forth get. Afterwards the different departments in the industry develop the single elements. The digital result of this phase is a CAD-model. In the assembly phase, which follows the design phase, it will be controlled if the CAD-models of the single elements are suitable. Until today the assembling is tested on physical mock-ups the most time. In this article only the digital assembling is of interest. The errors detected during the assembly phase have to be corrected in the following design phase. The design phase and assembly phase are interrelated to each other. Therefore it is important that an application for the installation and manipulation of wiring harness can be used in both the design phase and the assembly phase.

\section{$2.1 \quad$ The Design Phase}

During the design phase the two-dimensional plans for the cable installation are transformed by hand in a three dimensional model. Therefore CAD-Systems like CATIA [CATIA] or Adams [Adams] are used. In CATIA the cable shapes are calculated as splines. The cable length can be fixed and the maximal flexibility can be chosen. Also the start and the end orientation of the cables are adjustable. The disadvantage of the cable simulation module in CATIA is that not every combination of parameters (cables start and end-point, material components, etc.) produces a cable. If no cable could be created the user gets no hints, why the creation failed. Also the physically behaviour of the cable in the field of gravitation could not be simulated in CATIA. This missing physically behaviour, the non-intuitive usability and the sometime failed cable creations are the main disadvantages of the cable simulation in CATIA and perhaps in other CAD-systems, too. Another other kind of problem is that CATIA does not notice which cables are included in a harness. This missing information makes the manipulation of a harness very difficult.

During different interviews with persons, who are working in different departments of car construction, we had to learn that the exchange of datasets between different systems is most important. The best situation is 
that one can do all tasks in one system, because during every transformation of datasets information gets lost. Now the real advantage of the common CAD-systems is obvious. In the most CAD-systems one can do all tasks, which are needed during the construction phase in the same system. In most cases a constant dataset is more useful than a more efficient application.

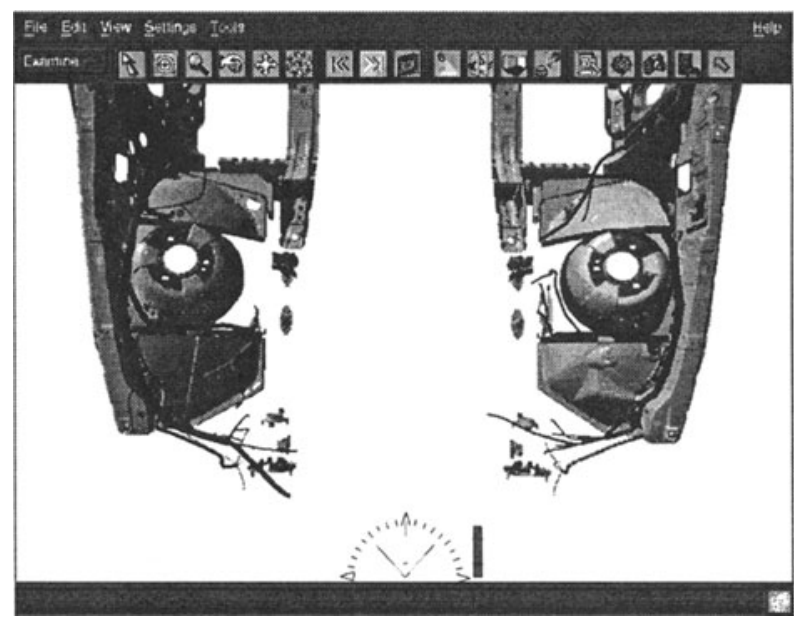

Figure 1. Cable installation with CATIA

\subsection{The Virtual Assembly Phase}

After the construction phase the virtual prototype has to be assembled. In this phase, the assembly phase, virtual reality applications are used. The CAD-dataset has to be converted in a dataset for a virtual reality (VR) application. In the VR application errors can be detected interactively. The most common kind of failure is the penetration, which is not visible in the CAD-system. Since a few years VR is used to detect if the designed parts match together. For example to test if a screw and a thread is suitable. After an error was found the CAD-model has to be modified. Of course here the data exchange could be also necessary, but until today the advantages of virtual reality applications are more useful than the advantage of a constant dataset. Actually none of the known VR-systems includes deformable cables, pipes and rubbers for the assembly test. 


\section{SYSTEM FOR THE INSTALLATION AND MANIPULATION OF WIRING HARNESS}

The advantage of the immersive 3D-environment (like CAVE or Powerwall) is that one can see the virtual product in its real size. In such an environment the virtual assembly tests can be performed in a very realistic manner. During the virtual assembly it should be tested if the designed elements match together and the best way for assemble different elements should be find out. Therefore the flexible elements like, cables, pipes or rubber plugs have to be pushed aside in a natural way.

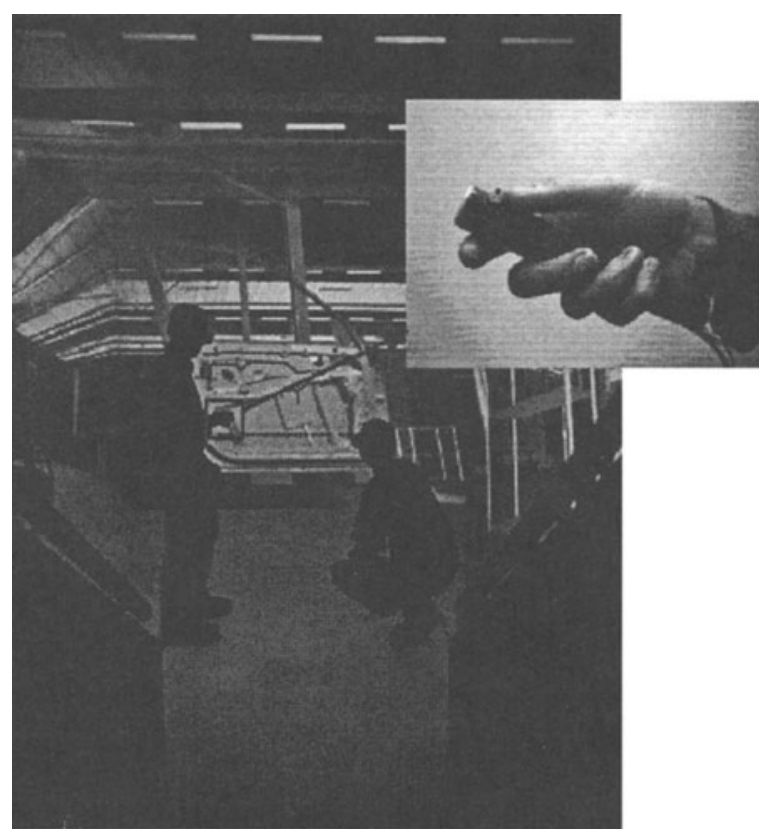

Figure 2. Cable manipulation with a Flystick as input device in the cave [IGD-CAVE].

To realize this prototypical application some developments were necessary. In this chapter the single developments are descript. We had to develop a cable simulation algorithm, a harness management, function selection and some interaction techniques and metaphors. After the chapter study you will see our goal was the implementation of an effective an easy usable. 


\subsection{The Cable Simulation}

The most important demands are visually correct results, real-time calculation and stable calculation behaviour. Another significant demand for a cable simulation used in an assembly application is the constant cable length during the simulation. If the cable is fixed on both ends then it should not get longer while the user pushes it aside. To fulfil these demands we developed a kinematics based cable simulation algorithm presented in [Hergen'00]. The bending property is also important in this context. The kinematical chain is complemented with mass points and rotation springs. Thereby the simulation of different materials is possible and consequentially a different bending property can be calculated. So the algorithm can simulate deformable, but inelastic and relatively stiff objects very well. It is a hierarchical method to ensure a real-time calculation on different processors. During the user interaction, a coarser resolution of the cable is simulated. If the user stops the cable movement, the resolution of the cable becomes refined, and so the surface becomes smoother.

\subsection{The Wiring Harness}

In reality, a vehicle needs a lot of cables and most of them are handled in a cable harness. So the next challenge of this application is the handling of the high complexity of the harness. To reduce the complexity of the cable harness for the simulation, we divide it into different cable sections.

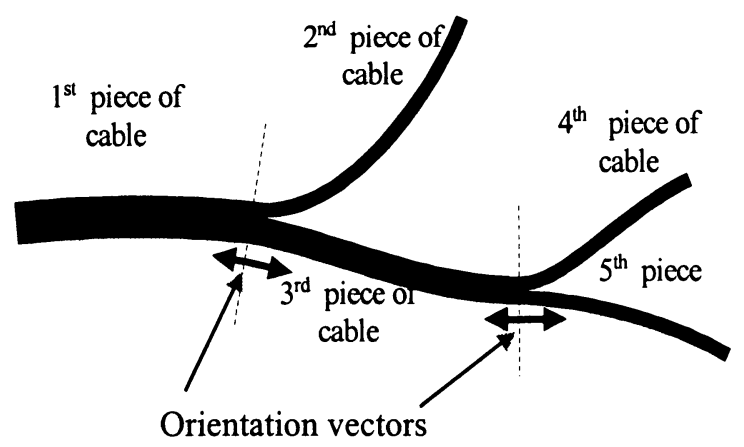

Figure 3. A wiring harness assembled from five sections 
In Figure 3 one can see an example of a divided harness. After the simulation of the individual harness pieces, the different cables should result in a smoothly coherent cable harness. We solved this problem using orientation vectors (Figure 3). They are placed at the start point and at the end point of every piece of cable and describe its orientation. It is also necessary to notice the cable, which build a harness. Our system memorizes the cable of a harness.

\subsection{Function Selection}

Also in our application we need the possibility to switch between various functions in a very simple and fast manner. In tests with different menu layouts it turns out that the pie menu [Callah'88] is the most suitable for our application. A pie menu is defined as a circle with 4 or 8 evenly distributed menu items (Figure 4). When opening the menu, the cursor is in the centre of the circle. The advantage of that type of menu is that all items are at the same distance from the initial position of the cursor.

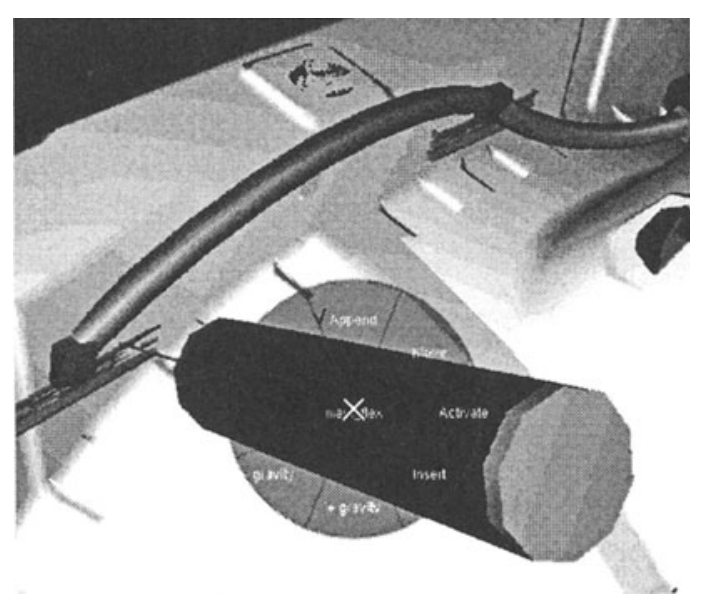

Figure 4. The pie menu

\subsection{Create a Harness With the Laser Beam Technique}

In this area of application the interaction with the single cables and with the whole cable harness is very important. To realize an effective input device, we adopt a well-known method for placing and selecting the cable's attachment points in the scene: the virtual laser beam [Bowman'97]. It is a very intuitive technique to select objects, which are not far away. The virtual 
beam is emitted from the hand in the direction the user points. An attachment point is selected if the beam penetrates it and the user triggers an event, like pressing a button on the input device.
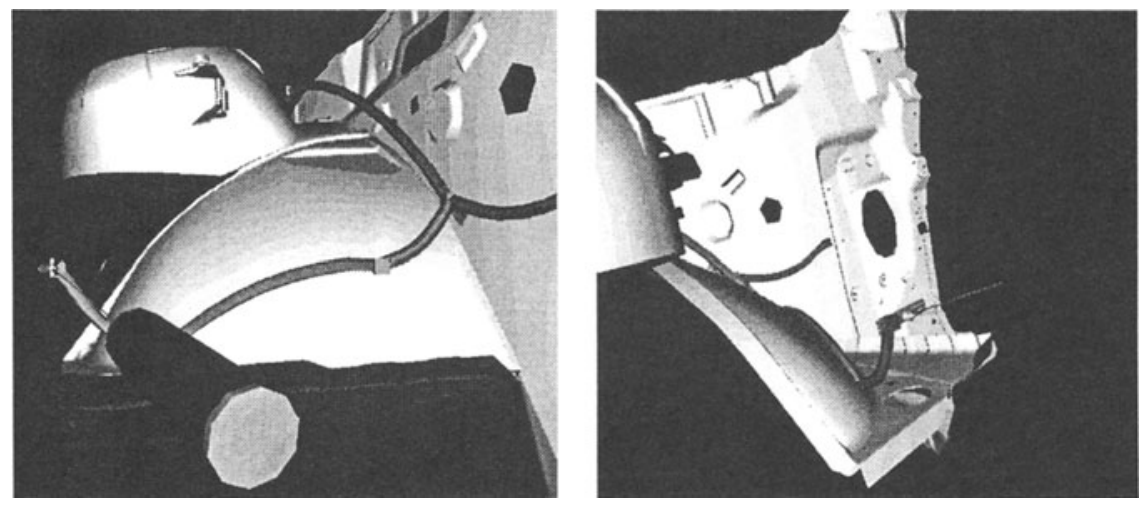

Figure 5. Cable installation in Virtual Environments from different points of view

\subsection{Virtual Reality Input Devices}

For immersive environments we use the Flystick [Knoepf'00] as input device. It is a tracked pen, equipped with three buttons. The position selection with the Flystick is intuitive and rather accurate. Desktop applications are more common than immersive environments are.

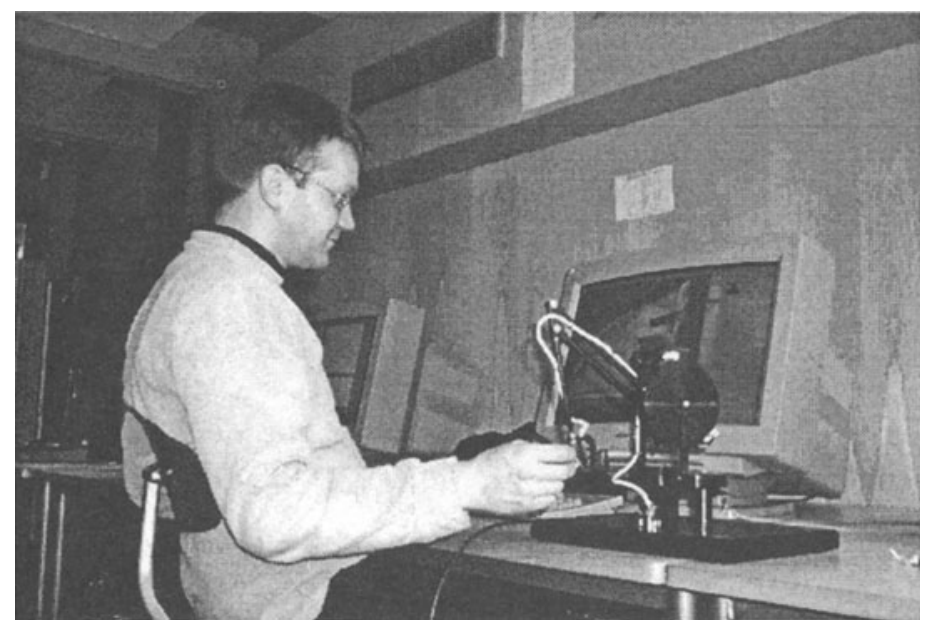

Figure 6. Using the Phantom as input device 
However, the problem of interaction in the 3D space remains. CAD applications offer the use of a spacemouse, a 2D mouse and a keyboard. Using a spacemouse is difficult for an untrained person. Therefore we search a more intuitive input device. We decided to test the application with the Phantom [Phantom]. The Phantom is an absolute input device for 3D desktop applications. It is a small robot arm with up to 6 degrees of freedom. The direct user input device is a pen with one button. In our tests we use the phantom without force feedback. They show that that the position selection is fast and quite precise.

\subsection{Interaction With Cables During the Assembly}

During the assembly phase the cables have to be pushed aside very often. Thus we had to realize this functionality in our application at first. In reality the user does this task mechanically, because it is so easy. But in VR-systems that task is not realized till this day. At first we use the laser beam method. Original, we used this method to position of the harness attachment points (chapter 3.4). Now we use the laser beam technique to push the cable aside. At first we have to select one attachment point and than the cable is pushed to the point where the beam penetrates an object. Using the beam, the cable could only move along another object. That is suitable during the installation phase, but during the assembly phase the cable should be moved freely. So the laser beam technique is unsuitable for the assembly phase.

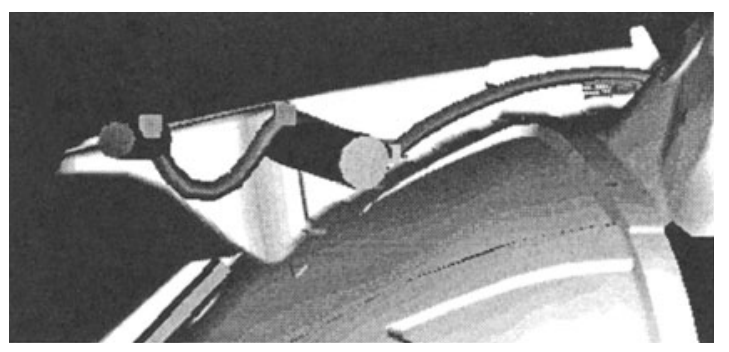

Figure 7. Moving the cable with the virtual copy of the Flystick

It makes more sense to push the cable away with the virtual copy of the input device. Figure 7 shows this operation. Pushing the cable aside by using the Flystick is very similar to pushing the cable aside by hand. Unhappily this method is unsuitable for a virtual assembly application. The reasons for this will be understandable after the explanation of the method's realization. If a collision between cable and virtual Flystick is recognized then the cable 
and the Flystick model are connected with each other on the collision point. If the user moves the Flystick then the cable moves, too. The problem is the connection of Flystick and cable. The cable glues on the Flystick, because the connection point cannot be changed during the interaction except the user triggers an event, which separates cable and Flystick. The steady connection has two disadvantages. The Flystick occludes the cable most of the time. The second disadvantage is the not existing visual feedback if the cable cannot be pushed or bowed any more. Without a visual feedback the user could not work with the application. The lack of user support is the reason why this method is not usable.

The next idea was to use of a second copy of the Flystick, the ghost. During a successful cable pushing the ghost is not visible. The ghost becomes only visible if the cable's movement stops. In this case the ghost represents the movement of the Flystick. This method is more effective than the former methods, but also with the ghost the user does not know why the cable does not move. To give the user a really informative feedback, we developed the cable ghost.

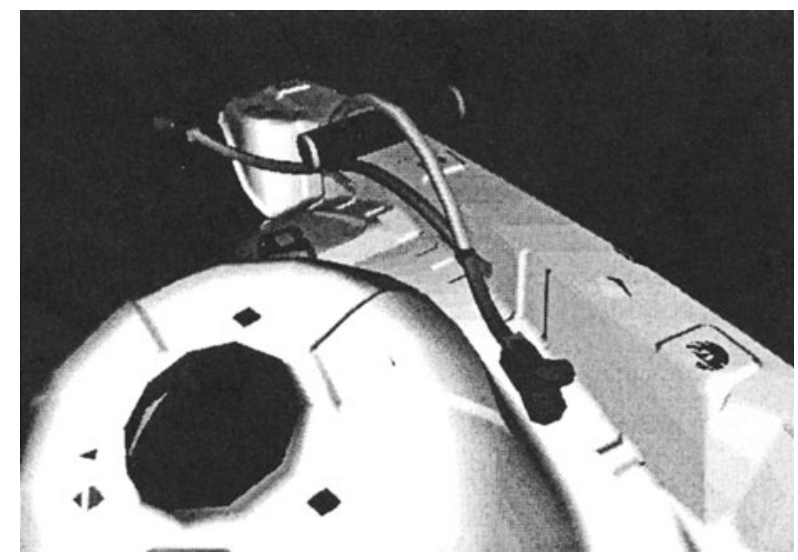

Figure 8. The cable ghost (green)

The principle is similar to the Flystick ghost. If the cable movement stops, because it cannot be stretched or bowed anymore, then the cable ghost becomes visible. The cable ghost can be realized as a spline or as an elastodynamical simulation. We chose a mass-spring simulation. On one hand the ghost cable can follow every movement of the Flystick, because it is highly deformable. On the other hand the kinematically simulated cable shows the realistic behaviour of the cable. Together both cable representations build a well usable and effective interface for the interaction with not elastically deformable objects. 


\subsection{Discussion of the Interaction Paradigms}

Our fundamental idea was the development of a user-friendly application. During the interaction the user should be supported as much as possible. This principle is reflected in all parts of the application also in the cable simulation itself. We made tests to choose the most accepted input device. It is the Flystick for immersive environments and the Phantom for desktop applications. The used interaction features like pie-menu or laser beam are accepted techniques. The use of a deformable ghost object is new in this type of application. The ghost object is highly deformable and can represent all user movements. The deformation of the ghost is the pattern for the deformation of the virtual cable model. This combination of the two cable representations provides the almost mechanical user interaction and the information about the real cable shape.

\section{CONCLUSION AND FUTURE WORK}

The digital development process in the car- and aircraft industry can be divided in two parts: the design phase, where the construction of the product is done, and the assembly phase, where the correction of the mock-up is done. The presented application has many functions, which are needed during the design phase [Hergen'01a][Hergen'01b]. The integration of haptical feedback has to be done in the future. This will make the cable installation faster and more accurate. Also the connection to a CAD-system is needed. At the moment we work on the expansion of the functionality for the assembly phase. The development of the cable ghost was a very important development step, because this paradigm enables an almost mechanical user interaction.

\section{REFERENCES}

[Adams] http://www.adams.com

[Bowman'97] D. A. Bowman, An Evaluation of Techniques for Grabbing and Manipulating Remote Objects in Immersive Virtual Environments: Symposium on Interactive 3D Graphics, Providence, USA, 1997, 35-38.

[Callah'88] J.Callahan, D. Hopkins, M. Weiser, B. Schneiderman, An empirical comparison of pie vs. linear menus, Proceedings of SIGCHI 1988, New York, USA, 1988, 95-100.

[CATIA] http://www.catia.com 
[Hergen'00] Hergenröther, E., Dähne P.: Real-Time Virtual Cables Based on Kinematics Simulation, The 8th International Conference in Central Europe on Computer Graphics, Visualization an Interactive Digital Media'2000, Plzen, Czech Republic, 2000, 402-409.

[Hergen'01a] Hergenröther, E., Knöpfle, C.: Cable Installation in Virtual Environments, Proceeding of the IASTED International Conference on Modelling and Simulation, Pittsburgh, USA, 2001, 276-280.

[Hergen'01b] Hergenröther, E., Knöpfle, C.: Installation and Manipulation of a Cable Harness in Virtual Environments, Proceeding of the IASTED International Conference on Robotics and Manufacturing, Cancun, Mexico, 2001, 240-244.

[IGD-CAVE] www.igd.fhg.de/igd-a4/index.html

[Knoepf'00] C. Knöpfle, G. Voß, An intuitive VR user interface for Design Review, Proceedings of Advanced Visual Interfaces 2000, Palermo, Italy, 2000, 98-103.

[Phantom] Developed by MIT Touch Lab: http://touchlab.mit.edu/ 